\title{
A novel hybrid intelligent fault diagnosis method based on improved RBF neural network
}

\author{
Liu Yi \\ School of Mechanical and Electronic Engineering \\ Wuhan Donghu University, Wuhan ,Hubei,430212, China \\ liuyi2396@163.com
}

\begin{abstract}
The radial basis function neural network (RBFNN) is a great potential artificial intelligence technology and can effectively realize the fault diagnosis for small sample and nonlinear problem. But the parameters of RBFNN model seriously affects the generalization ability and diagnosis accuracy on the great extent. So an improved differential evolution algorithm based on dynamic adaptive adjustment strategy is proposed to optimize the parameters of RBFNN model for obtaining the optimal RBFNN(DASDERBFNN) method. Then the proposed DASDERBFNN method is used to construct a new fault diagnosis (DSDRBFNFD) method. The experiment results show that the proposed DSDRBFNFD method can obtain the higher accuracy of fault diagnosis and is effective fault diagnosis for the engine.
\end{abstract}

Keywords:differential evolution, RBF neural network,intelligent fault diagnosis.

\section{Introduction}

With the rapid development of large-scale and complex modern industrial equipment, these equipment $\mathrm{s}$ will lead to increase the possibility of fault and maintenance, which brings great economic losses to enterprises. Therefore, it is very important to study the fault diagnosis technology.Fault diagnosis technology is a kind of state recognition technology based on the current status of the equipment and the historical status of the equipment. It is a state identification technology by using signal processing analysis method for evaluating the equipment status. The traditional fault diagnosis technology has a better effect on the analysis of the deep level fault of the complex structure, and the higher ability for the operator. In recent years, with the rapid development of artificial intelligence technology, the fault diagnosis technology is developed from the traditional technology to the intelligent technology[1,2]. The intelligent fault diagnosis technology is a kind of intelligent diagnosis methods based on knowledge processing technology. It can realize the integration of dialectical logic and mathematical logic, the unification of symbolic processing and numerical processing and reasoning process and algorithm process by the concept and process knowledge in order to realize the intelligent fault diagnosis method of equipment. The intelligent fault diagnosis method provides people with the powerful tool for solving the fault diagnosis problem of complex system[3-5].At present, a lot of scholars have studied the field of intelligent fault diagnosis and proposed a variety of intelligent fault diagnosis technologies[6-15].

Although these proposed fault diagnosis methods can better realize the fault diagnosis, but the accuracy of fault diagnosis is more lower. The RBFNN model can effectively realize the fault diagnosis for small sample and nonlinear problem. But the parameters of RBFNN model seriously affects the generalization ability and diagnosis accuracy. So an improved differential evolution algorithm based on dynamic adaptive adjustment strategy is proposed to optimize the parameters of RBFNN model for obtaining the optimal RBFNN(DASDERBFNN) method. Then the proposed DASDERBFNN method is used to construct a new fault diagnosis (DSDRBFNFD) method. Finally, the proposed DSDRBFNFD method is used to diagnose the fault of the cylinder of the engine in order to validate the diagnosis effectiveness of the DSDRBFNFD method. 


\section{RBF neural network}

Artificial neural network(ANN) is a great potential artificial intelligence technology, which is composed of a large number of nerve cells. The ANN provides the significant support for organizing, classifying, and summarizing data. It also helps to discern patterns among input data, require few assumptions, and achieve a high degree of accuracy for fault diagnosis. These advantages of ANN make the neural network technology with a potentially promising alternative tool for realizing the recognition, classification, and fault diagnosis with the accuracy, adaptability, robustness, effectiveness, and efficiency. The structure of ANN usually includes the input layer, hidden layer and output layer, shown in Figure 1.

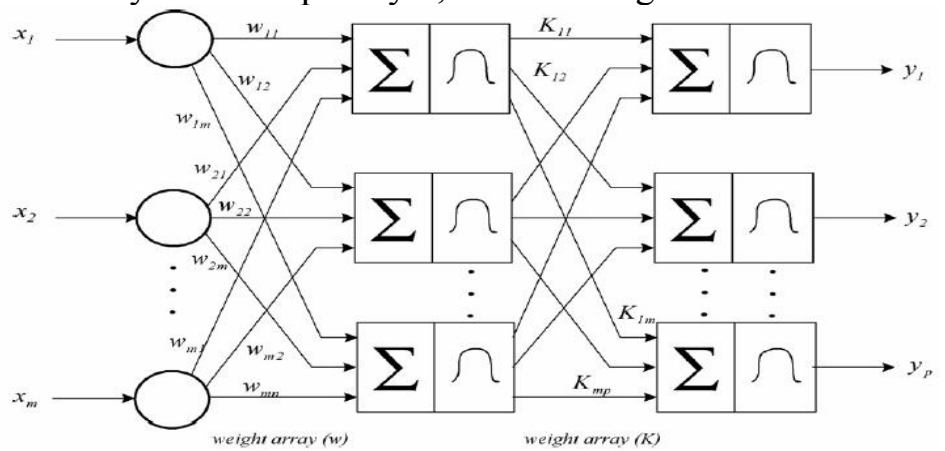

Figure 1. The structure of ANN

In the ANN, it contains very simple and highly interconnected processors, called neurons. The neurons are used to connect each other by the weighted links in order to transfer the signal from one layer to the other layer. The input layer is represented by circles and behaves as a buffer. Each neuron receives multiple inputs from other neurons, except for the neurons in the input layer, in proportion to their connection weights and then generates a single output in accordance with an activation function. An activation function can be linear or nonlinear form depending on applications. The ANN is trained in order to adjust the network weights by using the different learning algorithm. The training speed and real-time of the neural network are considered in order to be more adaptable for fault diagnosis. Because radial basis function neural network (RBFNN) takes on quick learning speed, so the RBFNN is selected to implement fault diagnosis in this paper. The RBFNN is to belong to the multi-layer forward neural network, shown in Figure 2.

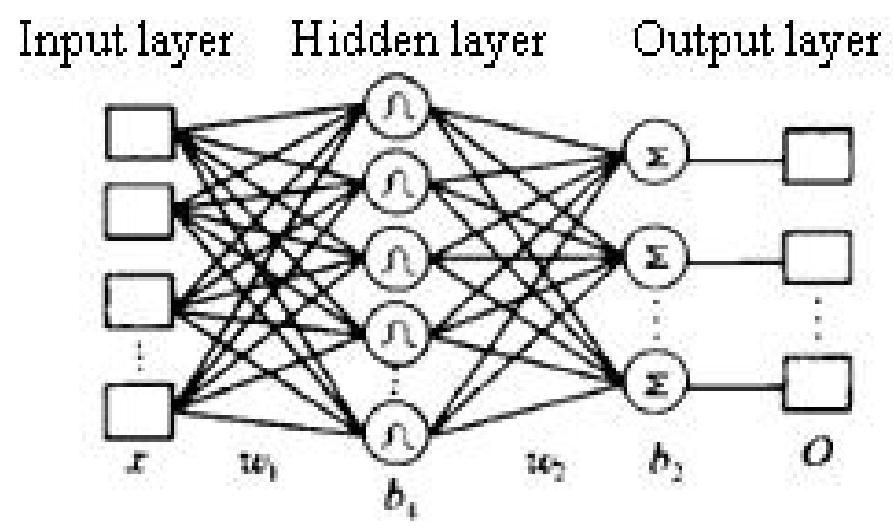

Figure 2. The structure of RBF neural network

As can be known from Figure 2, when the input learning vector of the RBFNN is $x=\{x, x 2, \ldots, x n\} T \in$ $R^{n}$, the output of the neuron $k$ in the output layer can be expressed as

$$
f(x)=\sum_{\mathbf{i}=\mathbf{1}}^{\mathrm{m}} w_{i} \phi\left(x, c_{i}\right)
$$

where $f(x)$ is output vector, $x$ is a $\mathrm{N}$-dimension input vector, $\phi\left(x, c_{i}\right)$ is a basis function and the output of the neuron $i$ in the hidden layer. $\phi$ is radial basis function, $c_{i}$ is the centre of the $i^{\text {th }}$ basis function with the same dimension as $x ; w=\left\{w_{1}, w_{2}, \ldots, w_{m}\right\}, T \in R^{m}$ is output weight matrix, $m$ is the number 
of the neuron in the input layer, $n$ is the number of the neuron in the hidden layer and $k$ is the number of the neuron in output layer. Gauss function is used to act the basis function, then

$$
\phi\left(x, c_{i}\right)=\exp \left(\frac{-\left\|x-c_{i}\right\|^{2}}{2 \sigma_{i}^{2}}\right)
$$

where $\sigma_{i}$ is the variance of Gauss samples. In the general, the learning algorithm of RBFNN uses a novel dynamic nearest neighbor-Clustering algorithm. The algorithm can generate the least nodes and high learning speed. So the key of the RBFNN is to determine the number of hidden nodes and width of corresponding center nodes.

\section{The optimization of RBFNN}

In the learning process of RBF neural network, three important parameters are determined. They are the center, the width and the weight of the network in the basis function of the hidden unit. At present, the number of hidden units is determined by using the experiences. However, the reasonable and accurate selection parameters can realize the nonlinear approximation ability of RBF neural network. The improved algorithm has strong optimization performance, it takes on the good robustness and parallel searching advantage. In order to obtain the better parameter optimization of the RBFNN, the optimism algorithm is used to optimize the parameters of the RBFNN in order to obtain the optimal RBFNN method. The selection of parameters in the RBFNN is regarded as a combination optimization of parameters in order to establish the objective function of combination optimization. The optimism algorithm is used to search for the optimal value of objective function. The optimization flow of RBFNN is described in Figure 3.

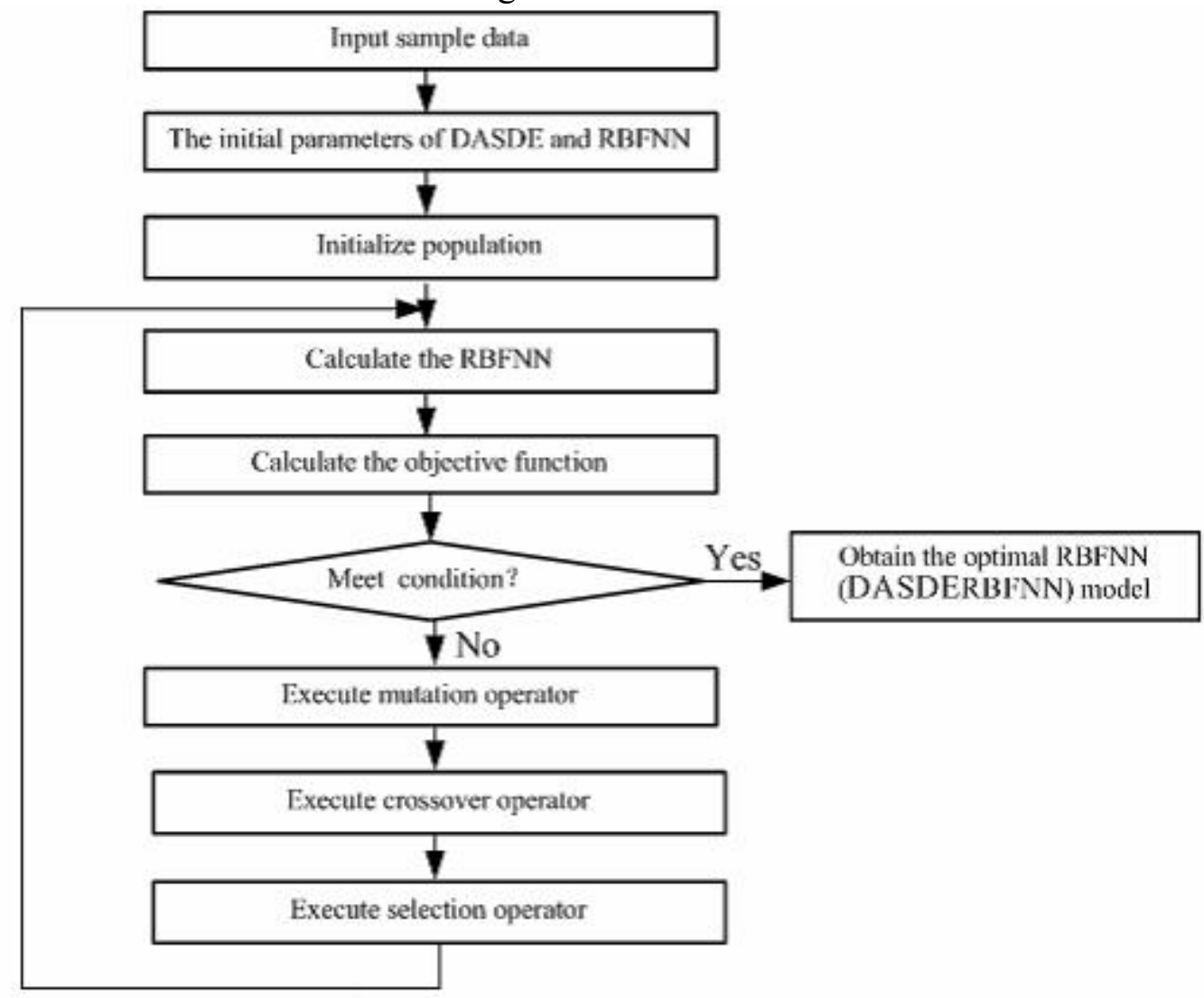

Figure 3. The flow of BFNN algorithm 


\section{A case analysis of the BFNFD method}

Engine is a kind of machine, which can transform other types of energy into mechanical energy. It includes gasoline engine, Sterling engine, steam engine, motor and so on. The engine is suitable for power generation device, and can also refer to the whole machine with power plant. In the operation process of the engine, the oil fault and gas fault are the most fault. Due to the complex structure of the engine, it is difficult to distinguish the causes of the fault. So the proposed BFNFD method is used to achieve the fault diagnosis of the engine.

In the operation process of the engine, six characteristic parameters are often selected. These characteristic parameters are the maximum acceleration index(AR), the average acceleration index(MC), the maximum deceleration index(DR), the average deceleration index(MI), the torque harmonic component ratio(TA) and the rising speed of the gas explosion(PI). When the fault diagnosis is diagnosed, the characteristic parameters are extracted at the first. The cylinder of the engine has three faults and one normal state. Three faults are the less oil, valve leakage and cylinder leakage. The four sample data are used to test the DSDRBFNFD method, shown in Table 1. These sample data are not very different. The DSDRBFNFD method is initialized as follows: the input unit number is 6 , the output variable is 4,population size is $N P=100$, scaling factor is $F=0.7$, the number of iteration is $T=500$. The initial crossover probability is $C R_{\min }=0.50$ and $C R_{\max }=1.0$. The BFNFD method is independently run 30 times. The initial decay rate and the initial growth rate are 80. The environment is: Matlab 2012a, running on the Pentium IV, 2.0GB RAM..

Table 1. The sample data for testing

\begin{tabular}{cccccccc} 
Index & Cylinder state & AR & MC & DR & MI) & TA & PI \\
\hline 1 & Less oil & 0.112 & 0.104 & 0.034 & 0.089 & 0.083 & 0.098 \\
2 & Valve leakage & 0.114 & 0.131 & 0.019 & 0.084 & 0.122 & 0.199 \\
3 & Cylinder leakage & 0.225 & 0.193 & 0.145 & 0.104 & 0.121 & 0.111 \\
4 & Normal state & 1.002 & 1.003 & 0.998 & 1.002 & 1.005 & 0.998
\end{tabular}

The test sample data in Table 1 are executed in order to obtain the fault diagnosis results by using the proposed BFNFD method for one time, shown in Table 2.

Table 2. The diagnosis result of the proposed BFNFD method

\begin{tabular}{|c|c|c|c|c|c|c|}
\hline Index & Cylinder state & & Dutput & he result & & Diagnosis result \\
\hline 1 & Less oil & 0.006 & 0.009 & 0.924 & 0.005 & Less oil \\
\hline 2 & Valve leakage & 0.007 & 0.031 & 0.007 & 0.915 & Valve leakage \\
\hline 3 & Cylinder leakage & 0.001 & 0.903 & 0.004 & 0.006 & Cylinder leakage \\
\hline 4 & Normal state & 1.001 & 0.073 & 0.0215 & 0.008 & Normal \\
\hline
\end{tabular}

\section{Conclusions}

The RBFNN model is a great potential artificial intelligence technology with a large number of nerve cells. It can organize, classify, and summarize the data. And It can effectively realize the fault diagnosis for small sample and nonlinear problem. But the parameters of RBFNN model seriously affects the generalization ability and prediction accuracy on the great extent. So an improved differential evolution algorithm based on dynamic adaptive adjustment strategy is proposed to optimize the parameters of RBFNN model for obtaining the optimal RBFNN method. The result shows that the optimized RBF neural network based on improved DE algorithm can improve the accuracy of diagnosis. 


\section{Acknowledgement}

This work is supported by the Youth fund of Wuhan Donghu University.

\section{References}

[1] W. Deng, R. Chen,B. He, Y.Q. Liu, L.F. Yin, J.H. Guo, "A novel two-stage hybrid swarm intelligence optimization algorithm and application", Soft Computing, vol.16,no.10, (2012),pp.1707-1722.

[2] W. Deng, R. Chen, J. Gao, Y.J. Song, J.J. Xu, "A novel parallel hybrid intelligence optimization algorithm for a function approximation problem", Computers and Mathematics with Applications, vol.63, no.1, (2012), pp. 325-336.

[3] W . Deng, H.M. Zhao, J.J. Liu, X.L. Yan, Y.Y. Li, L.F. Yin, C.H. Ding, “An improved CACO algorithm based on adaptive method and multi-variant strategies", Soft Computing, vol.19,no.3,(2015),pp.701-713.

[4] K. Guo,Y. Zhu,Y. San, "Analog circuit intelligent fault diagnosis based on greedy KPCA and one-against-rest SVM approach", Journal of Theoretical and Applied Information Technology, vol.46,no.1, (2012), pp. 147-157.

[5] L. Xiang and W. Cui, "Study of intelligence diagnosis system for wind turbine gearbox fault", Telkomnika - Indonesian Journal of Electrical Engineering, vol.11,no.8, (2013), pp. 4691-4697.

[6] W. Deng,X.H. Yang, J.J. Liu, H.M. Zhao,Z.G. Li, X.L. Yan, "A novel fault analysis and diagnosis method based on combining computational intelligence methods", Proceedings of the Institution of Mechanical Engineers, Part E: Journal of Process Mechanical Engineering, vol.227,no.3, (2013), pp. 198-210.

[7] A. Krishnakumari and A. Elayaperumal, "Gear fault diagnosis using vibration signals based on decision tree assisted intelligent controllers", Journal of Vibroengineering, vol.15,no.4, (2013), pp. 1826-1837.

[8] Y.S. Wang, Q.H. Ma, Q. Zhu, X.T. Liu, L.H. Zhao, “An intelligent approach for engine fault diagnosis based on Hilbert-Huang transform and support vector machine", Applied Acoustics, vol.75,no.1, (2014),

[9] W.L. Shang, X.F. Zhou, J. Yuan, "An intelligent fault diagnosis system for newly assembled transmission”, Expert Systems with Applications, vol.41,no.9, (2014), pp. 4060-4072.

[10]J.H. Zhang, W.P. Ma, J.W. Lin,L. Ma,X.J. Jia, "Fault diagnosis approach for rotating machinery based on dynamic model and computational intelligence", Measurement: Journal of the International Measurement Confederation, vol.59,no.1, (2015), pp. 73-87.

[11]X.L. Zhang, B.J. Wang, X.F. Chen, "Intelligent fault diagnosis of roller bearings with multivariable ensemble-based incremental support vector machine", Knowledge-Based Systems, vol.89, (2015), pp. 56-85.

[12]L. Aydn, M. Karaköse, E. Akn, "Combined intelligent methods based on wireless sensor networks for condition monitoring and fault diagnosis", Journal of Intelligent Manufacturing, vol.26,no.4, (2015), pp. 717-729.

[13]R. Gopinath,C. Santhosh Kumar,K.I. Ramachandran, V. Upendranath, P.V.R. Sai Kiran, "Intelligent fault diagnosis of synchronous generators", Expert Systems with Applications, vol.45,no..3, (2016), pp. 142-149.

[14]H. Zou and F.Z. Huang, "A novel intelligent fault diagnosis method for electrical equipment using infrared thermography”, Infrared Physics and Technology, vol.73, (2015), pp. 29-35.

[15]J.X. Qu, Z.S. Zhang,T. Gong, "A novel intelligent method for mechanical fault diagnosis based on dual-tree complex wavelet packet transform and multiple classifier fusion", Neurocomputing, vol.171, (2016),837-853. 